\title{
Human Factors and Ergonomics in the Design of Health Information Technology: Trends and Progress in 2014
}

\author{
S. Pelayo ${ }^{1}$, MS. Ong ${ }^{2,3}$, Section Editors for the IMIA Yearbook Section on Human Factors and \\ Organizational Issues \\ 1 INSERM CIC-IT 1403 Evalab, CHU Lille, UDSL EA 2694, Lille University, Lille, France \\ 2 Children's Hospital Informatics Program, Boston Children's Hospital, Boston MA 02115, United States \\ 3 Center for Health Informatics, Australian Institute of Health Innovation, Macquarie University, \\ Sydney, Australia
}

\begin{abstract}
Summary
Objective: To summarize significant contributions to the research on human factors and organizational issues in medical informatics.

Methods: An extensive search using PubMed/Medline and Web of Science $®$ was conducted to identify the scientific contributions, published in 2014, to human factors and organizational issues in medical informatics, with a focus on health information technology (HIT) usability. The selection process comprised three steps: (i) 15 candidate best papers were selected by the two section editors, (ii) external reviewers from a pool of international experts reviewed each candidate best paper, and (iii) the final selection of three best papers was made by the editorial board of the IMIA Yearbook.

Results: Noteworthy papers published in 2014 describe an efficient, easy to implement, and useful process for detecting and mitigating human factors and ergonomics (HFE) issues of HIT. They contribute to promote the HFE approach with interventions based on rigorous and well-conducted methods when designing and implementing HIT.

Conclusion: The application of HFE in the design and implementation of HIT remains limited, and the impact of incorporating HFE principles on patient safety is understudied. Future works should be conducted to advance this field of research, so that the safety and quality of patient care are not compromised by the increasing adoption of HIT.
\end{abstract}

\section{Keywords}

Human factors, health information technology, patient safety

Yearb Med Inform 2015;10:75-8

http://dx.doi.org/10.15265/IY-2015-033

Published online August 13, 2015

\section{Introduction}

While health information technology (HIT) promises to enhance patient care quality, safety, and efficiency, significant concerns about its unintended consequences have been raised [1-3]. The lack of consideration for human factors and ergonomics (HFE) ${ }^{1}$ in the design of a system is now recognized as a critical barrier to the success of HIT [5-8]. As a result, the Institute of Medicine (IOM) has called, in a 2012 report, for a more effective integration of HFE approaches in the design, evaluation, and implementation of HIT in clinical settings [9].

HFE refers to the application of knowledge about human capabilities (physical, sensory, emotional, and intellectual), and limitations, to the design and development of a system [10]. Usability is one specific dimension of HFE: "the extent to which a product can be used by specified users to achieve specified goals with effectiveness, efficiency and satisfaction in a specified context of use" [11]. Usability goes beyond the features of the interface (e.g. legibility of texts, layout or prompting) to address the compatibility of a system behavior with users' needs [12]. This approach considers human-machine mismatches as one basis of human errors, and emphasizes the importance of user-centric design to guarantee the over-

\footnotetext{
Human Factors and Ergonomics are considered as synonymous for the discipline; the discipline is often referred to as "Human Factors and Ergonomics" or HFE [4].
}

all performance and safety of a system [13]. Such mismatches can stem from improper interface design, to the lack of knowledge of users' preferences, clinicians' workflow, and information need. The identification of key usability features and functions of HIT related to users' capabilities, limitations, and needs should also be considered to design usable and safe systems. The violation of these design principles may lead to systems prone to usage errors, which can adversely affect patient care quality [14-15].

Despite the growing awareness of the importance of HFE in the design of HIT systems, application of HFE in healthcare remains "in its infancy" [16]. While the earliest reported study of HFE in healthcare dated back several decades [17], HFE-based interventions are still far from being systematically performed or, at best, their adoption and use are incomplete [16]. Methods for incorporating HFE design principles into clinical settings, and their impact on patient safety, remain under-studied [18-19]. Efforts towards considering HFE approaches should be made through the use of specific or additional methods. Further efforts in HFE research are still needed to think and plan the logistics of HFE use.

In 2015, the selection of papers in the Human Factors and Organizational Issues (HFOI) section intends to illuminate current progress of HFE research in medical informatics. Specifically, we seek to identify innovative and/or effective HFE methods for supporting the identification, 
prevention and/or mitigation of HFE/ usability concerns during the design and implementation of HIT.

\section{Selection of Best Papers}

The detailed description of the paper selection process has been described elsewhere [19]. In brief, two electronic databases were searched, PubMed/Medline and Web of Science ${ }^{\circledR}$. Searches were performed in November and December 2014 to identify peer-reviewed journal articles published in 2014, in the English language, and related to HFE research in medical informatics. In addition to the search of electronic databases, manual searches of key themes were performed in major biomedical journals (e.g. Journal of the American Medical Informatics Association, Methods of information in medicine, Journal of medical Internet research, etc.).

Used keywords included both free-text and coded keywords. Free-text keywords were listed as regards to the questions addressed by the section. Corresponding relevant MeSH terms were identified. Pubmed was queried to test keywords in an iterative process. Consequently, two queries were built: one based on MeSH terms used as major topic in the Pubmed/ Medline database, the second one based on free-text keywords searched in title or abstracts through Pubmed/Medline and Web of Science ${ }^{\circledR}$ databases.

One of the two section editors performed the searches. Database searches yielded 1,132 papers, and the manual searches identified 202 additional papers, giving a total of 1,334 references. The two section editors undertook independently the first screening of titles and abstracts to identify papers relevant to the field of interest. The two section editors classified the papers into three categories: accepted, rejected, or pending. They then reviewed in detail the accepted and pending full-text articles to finally reach a consensual list of 15 candidate best papers. Two papers were already selected in two other sections [20-21], and were removed from the HFOI selection, because considered to be better relevant to these sections. Papers were

Table 1 Best paper selection of articles for the IMIA Yearbook of Medical Informatics 2015 in the section 'Human Factors and Organizational Issues'. The articles are listed in alphabetical order of the first author's surname.

Section

Human Factors and Organizational Issues

- Flewwelling CJ, Easty AC, Vicente KJ, Cafazzo JA. The use of fault reporting of medical equipment to identify latent design flaws. J Biomed Inform 2014 0ct;51:80-5.

- Friedman A, Crosson JC, Howard J, Clark EC, Pellerano M, Karsh BT, Crabtree B, Jaén CR, Cohen DJ. A typology of electronic health record workarounds in small-to-medium size primary care practices. J Am Med Inform Assoc 2014 Feb;21(el):e78-83.

- Russ AL, Zillich AJ, Melton BL, Russell SA, Chen S, Spina JR, Weiner M, Johnson EG, Daggy JK, McManus MS, Hawsey JM, Puleo AG, Doebbeling BN, Saleem JJ. Applying human factors principles to alert design increases efficiency and reduces prescribing errors in a scenario-based simulation. J Am Med Inform Assoc 2014 0ct;21(e2):e287-96.

considered according to their originality, innovativeness, scientific quality, and scientific and/or practical impact..

In agreement with the IMIA Yearbook process, the 13 finally selected candidate best papers were evaluated by the two section editors, by IMIA Yearbook editors, and by additional external reviewers (at least four reviewers per paper). Three papers were finally selected as best papers (Table 1). A content summary of the selected best papers can be found in the appendix of this synopsis.

\section{Outlook of Selected Papers and Conclusions}

Noteworthy papers published in 2014 design an efficient, easy to implement, and useful process for detecting and mitigating HFE problems of technologies. By leveraging maintenance data that are routinely collected, Flewwelling et al. [22] present a cost-effective and efficient method for detecting devices that may be poorly designed. Friedman et al. [23] give a general framework for EHR users to identify and address workarounds, and for researchers to examine the effect of different types of EHR workarounds on patient safety, care quality, and efficiency. Russ et al. [24] provide some of the first experimental evidence about the specific aspects of the HFE-based redesign that are likely to contribute to patient safety. The study strongly contributes to the efforts that should be made to support HFE-based interventions through the evidence for their effectiveness.
The other candidate best papers contribute to promote the HFE approach with interventions based on rigorous and well-conducted methods when designing and implementing HIT. Some of them convincingly emphasize the value of clinical-based assessment to compensate the insufficiency of the hospital traditional ways of assessing IT systems [25-26]. Other papers illustrate the importance of complementary validated measures, each one providing unique insights to specific usability challenges and problems [27-28]. Phansalkar et al. [29] show the usefulness of HFE design principles to design safe and usable HIT relying on a substantial well-detailed and illustrated evaluation.

The remaining selected papers focus on the challenges posed by $\mathrm{m}$-Health products and/or technologies in the homecare setting. The number of papers addressing these issues increases in 2014. The design and evaluation of this kind of HIT have become a challenge for ensuring the patient safety and the effectiveness of the systems. The spate of health-related apps may lead consumers, patients, and caregivers to use interventions that lack evidence of efficacy [30]. The selected studies point out the critical aspects to consider in this field. In a systematic review, Peek et al [31] highlight the need for considering the factors of acceptance of electronic technologies, especially by the current generation of seniors. Guise et al. [32] conclude that the home environment is complex to analyze. Patient safety issues are embedded within overarching cultural, social and political structures and circumstances that govern healthcare in the home environment. Mirkovic et al. [33] demonstrate 
the numerous challenges when designing a mobile platform. They even define a set of general design recommendations that can be used when developing patient support mobile apps with similar design and functionality requirements.

\section{Acknowledgement}

We would like to thank Martina Hutter for her support and the reviewers for their participation in the selection process of the IMIA Yearbook.

\section{References}

1. Berg M, Coiera E. Some unintended consequences of information technology in health care: the nature of patient care information system-related errors. J Am Med Inform Assoc 2004 MarApr;11(2):104-12.

2. Magrabi F, Ong MS, Runciman B, Coiera E. Using FDA reports to inform a classification for health information technology safety problems. J Am Med Inform Assoc 2012;19(1):45-53.

3. Magrabi F, Ong MS, Runciman B, Coiera E. An analysis of computer-related patient safety incidents to inform the development of a classification. J Am Med Inform Assoc 2010;17(6):663-670.

4. Carayon P, Xie A, Kianfar S. Human factors and ergonomics as a patient safety practice. BMJ Qual Saf 2014 Mar;23(3):196-205.

5. Institute of Medicine (IOM). Patient safety: achieving a new standard for care. National Academies Press; 2003.

6. Patel VL, Zhang J, Yoskowitz NA, Green R, Sayan OR. Translational cognition for decision support in critical care environments: a review. J Biomed Inform 2008 Jun;41(3):413-31.

7. Zhang J. Human-centered computing in health information systems. Part 1: analysis and design. J Biomed Inform 2005 Feb;38(1):1-3.

8. Zhang J. Human-centered computing in health information systems part 2: evaluation. J Biomed Inform 2005 Jun;38(3):173-5.

9. Institute of Medicine (IOM). Health IT and Patient Safety: Building Safer Systems for Better Care. Washington, DC: Institute of Medicine; 2011.

10. ANSI/AAMI HE75:2009. Human factors engineering - Design of medical devices. Arlington, VA.

11. International Standardization Organization. Ergonomic requirements for office work with visual display terminals (VDTs) -- Part 11: Guidance on usability (Rep N 9241-11). Geneva: International Standardization Organization; 1998.

12. Beuscart-Zéphir MC, Borycki E, Carayon P, Jaspers MW, Pelayo S. Evolution of human factors research and studies of health information technologies: the role of patient safety. Yearb Med Inform 2013;8(1):67-77.

13. International Organisation for Standardization, ISO 9241-210:2010 Ergonomics of human-system interaction - Part 210: Human centred design for interactive systems, Geneva; 2010.

14. Marcilly R, Beuscart-Zéphir MC, Ammenwerth E, Pelayo S. Seeking evidence to support usability principles for medication-related clinical decision support (CDS) functions. Stud Health Technol Inform 2013;192:427-31.

15. Marcilly R, Boog C, Leroy N, Pelayo S. Perceived usefulness of a usability issues reporting form to help understand "usability-induced use-errors": a preliminary study. Stud Health Technol Inform 2014;205:880-4.

16. Patel VL, Kannampallil TG. Human factors and health information technology: current challenges and future directions. Yearb Med Inform 2014 Aug 15;9(1):58-66.

17. Safren MA, Chapanis A. A critical incident study of hospital medication errors. Hospitals 1960 May 1;34:32-4; passim.

18. Saleem JJ, Russ AL, Sanderson P, Johnson TR, Zhang J, Sittig DF. Current challenges and opportunities for better integration of human factors research with development of clinical information systems. Yearb Med Inform 2009:48-58.

19. Lamy JB, Séroussi B, Griffon N, Kerdelhué G, Jaulent MC, Bouaud J. Toward a formalization of the process to select IMIA Yearbook best papers. Methods Inf Med 2015;54(2):135-44.

20. Meeks DW, Smith MW, Taylor L, Sittig DF, Scott JM, Singh H. An analysis of electronic health record-related patient safety concerns. J Am Med Inform Assoc 2014 Nov-Dec;21(6):1053-9.

21. Goddard K, Roudsari A, Wyatt JC. Automation bias: empirical results assessing influencing factors. Int J Med Inform 2014 May;83(5):368-75.

22. Flewwelling CJ, Easty AC, Vicente KJ, Cafazzo JA. The use of fault reporting of medical equipment to identify latent design flaws. J Biomed Inform 2014 Oct;51:80-5.

23. Friedman A, Crosson JC, Howard J, Clark EC, Pellerano M, Karsh BT, et al. A typology of electronic health record workarounds in small-to-medium size primary care practices. J Am Med Inform Assoc 2014 Feb;21(e1):e78-83.

24. Russ AL, Zillich AJ, Melton BL, Russell SA, Chen $\mathrm{S}$, Spina JR, et al. Applying human factors principles to alert design increases efficiency and reduces prescribing errors in a scenario-based simulation. J Am Med Inform Assoc 2014 Oct;21(e2):e287-96.

25. Jensen S, Rasmussen SL, Lyng KM. Evaluation of a clinical simulation-based assessment method for EHR-platforms. Stud Health Technol Inform 2014;205:925-9.

26. Jorritsma W, Cnossen F, van Ooijen PM. Merits of usability testing for PACS selection. Int J Med Inform 2014 Jan;83(1):27-36.

27. Walji MF, Kalenderian E, Piotrowski M, Tran D, Kookal KK, Tokede O, et al. Are three methods better than one? A comparative assessment of usability evaluation methods in an EHR. Int J Med Inform 2014 May;83(5):361-7.

28. Moore LJ, Wilson MR, McGrath JS, Waine E, Masters RS, Vine SJ. Surgeons' display reduced mental effort and workload while performing robotically assisted surgical tasks, when compared to conventional laparoscopy. Surg Endosc 2014 Nov 27. [Epub ahead of print]

29. Phansalkar S, Zachariah M, Seidling HM, Mendes C, Volk L, Bates DW. Evaluation of medication alerts in electronic health records for compliance with human factors principles. J Am Med Inform Assoc 2014 Oct;21(e2):e332-40.

30. Baker TB, Gustafson DH, Shah D. How can research keep up with eHealth? Ten strategies for increasing the timeliness and usefulness of eHealth research. J Med Internet Res 2014 Feb 19;16(2):e36.

31. Peek ST, Wouters EJ, van Hoof J, Luijkx KG, Boeije HR, Vrijhoef HJ. Factors influencing acceptance of technology for aging in place: a systematic review. Int J Med Inform 2014 Apr;83(4):235-48.

32. Guise V, Anderson J, Wiig S. Patient safety risks associated with telecare: a systematic review and narrative synthesis of the literature. BMC Health Serv Res 2014 Nov 25;14(1):588.

33. Mirkovic J, Kaufman DR, Ruland CM. Supporting cancer patients in illness management: usability evaluation of a mobile app. JMIR Mhealth Uhealth 2014 Aug 13;2(3):e33.

\section{Correspondence to:}

Sylvia Pelayo

INSERM CIC-IT 1403

Université Lille 2, CHRU de Lille

Lille, France

E-mail: sylvia.pelayo@univ-lille2.fr

\section{Content Summaries of Best Papers Selected for the 2015 Edition of the IMIA Yearbook, Section HFOI}

\author{
Flewwelling CJ, Easty AC, Vicente KJ, \\ Cafazzo JA \\ The use of fault reporting of medical \\ equipment to identify latent design flaws
}

\section{J Biomed Inform 2014;51:80-5}

Flewwelling et al. introduced an innovative approach to identifying devices with a high potential of containing latent usability-related design flaws. At the study site, a "No Fault Found" (NFF) incident is reported whenever a medical device sent to the hospital engineering service department for troubleshooting is found to be operating as intended. The authors hypothesized that these incidents may reflect usability-related design flaws or intermittent failures that warrant further investigation. The authors conducted an analysis of the medical equipment maintenance data collected at the hospital. They demonstrated that NFF reporting frequency correlated strongly 
with the usability of a device. Thus, by monitoring NFF reporting rates, poorly designed devices that may adversely affect clinical workflow can be identified.

Russ AL, Zillich AJ, Melton BL, Russell SA, Chen S, Spina JR, Weiner M, Johnson EG, Daggy JK, McManus MS, Hawsey JM, Puleo AG, Doebbeling BN, Saleem JJ

Applying human factors principles to alert design increases efficiency and reduces prescribing errors in a scenario-based simulation

\section{J Am Med Inform Assoc 2014;21:e287-e296}

Russ et al. provided some of the first experimental evidence for the impact of HFE on patient safety. Specifically, the study supports the hypothesis that incorporating human factors principles to the interface design of medication alerts improved usability, perceived workload, and prescribing errors. Aspects of the redesigned alerts that likely contribute to better prescribing include the provision of clinical data closer to the point of decision, and the display of alerts in a tabular format. This research also identified some features, such as scrolling, that pose high patient safety risks. The study clearly demonstrates that simple design changes could have significant impact on the safety and efficacy of HIT systems. Similarly, overlooking the usability issues that may appear trivial can have detrimental effects on patient safety.

\section{Friedman A, Crosson JC, Howard J, Clark EC, Pellerano M, Karsh Ben-Tzion, Crabtree $B$, Jaen CR, Cohen DJ \\ A typology of electronic health record workarounds in small-to-medium size primary care practices}

\section{J Am Med Inform Assoc 2014;21:e78-e83}

Friedman et al. conceptualized a general framework based on a workaround typology to assess the impact of electronic health records (EHRs) on clinical workflows and work activities. "Workarounds" are defined as behaviors users adopt to overcome the perceived limitations of a technical system. Workarounds may represent undesirable deviations from the intended use of a technology. However, workarounds are not inherently negative and can be useful signals of misalignment between work tasks and the HIT system being used, pointing to needed change. The authors used direct observations to construct a typology of workarounds that identifies conceptual features of workarounds, including characteristics that distinguish benign or positive workarounds from those that are potentially harmful. To date, there have been few attempts to categorize workarounds in general. This typology begins the important work of separating workarounds that are benign or even beneficial from those that are more concerning by drawing attention to three key questions: is the workaround avoidable? Is the workaround temporary or has it become incorporated into the normal workflow? Was the workaround explicitly chosen as an improvement or was it unplanned? Categorizing user-generated workarounds is an important first step toward creating the awareness of workarounds, understanding the implications of these behaviors in practice, and eliminating unwanted or unsuccessful workarounds. 